International Journal of Advanced Biological and Biomedical Research Available online at http:www.ijabbr.com

Volume 8, Issue 1 (2020) pp. 9-16

DOI: 10.33945/SAMI/IJABBR.2020.1.2

Research Article

\title{
Soybean Seeds Treated with Single Walled Carbon Naotubes (SwCNTs) Showed Enhanced Drought Tolerance During Germination
}

\section{Sun Wenli ${ }^{1,2}$, Mohamad Hesam Shahrajabiann ${ }^{1,2, *}$, Qiman Huang ${ }^{1}$}

\author{
${ }^{1}$ Biotechnology Research Institute, Chinese Academy of Agricultural Sciences, Beijing \\ 100081, China \\ ${ }^{2}$ Nitrogen Fixation Laboratory, Qi Institute, Building C4, No. 555 Chuangye Road, Jiaxing \\ 314000, Zhejiang, China
}

*Corresponding Author E-mail: hesamshahrajabian@gmail.com

Received: 25 May 2019, Revised: 28 June 2019, Accepted: 10 August 2019

\begin{abstract}
Drought cause yield loss of soybean production is the major reason to yield losing of soybean. To improve the germination and growth of soybean seeds under drought stress. We studied the effect of the single walled carbon nanotubes (SwCNTs) material in seed germination and the physiological changes occurred under drought condition, performed SwCNTs treatments in soybean (Zhonghuang 35) seeds at PEG drought stress, evaluated the germination, root and shoot length, fresh weight, the activities of superoxide dismutase (SOD), peroxidase (POD), catalase (CAT), and the contents of maleic dialdehyde (MDA), ascorbic acid (AsA) and $\mathrm{H}_{2} \mathrm{O}_{2}$. The results showed that under osmotic potentials of $0,-0.3,-0.6$ with PEG 6000, the germination percentage of SwCNTs-treated seeds was higher than the control treatment (SwCNTs-untreated seeds). The fresh weight of SwCNTs-treated seeds were evidently higher than that of the controls under the osmotic potentials of $0,-0.3,-0.6 \mathrm{MPa}$. The root and shoot length were also longer than control in SwCNTs-treated seeds at 0, $-0.3,-0.6 \mathrm{MPa}(\mathrm{P}<0.05, \mathrm{P}<0.01)$ and $-0.6 \mathrm{MPa}(\mathrm{P}<0.01)$ respectively. Moreover, the MDA and $\mathrm{H}_{2} \mathrm{O}_{2}$ contents were lower but ASA content and activities of SOD, CAT, POD were higher in SwCNTs-treated seeds than control treatment under osmotic potentials of $-0.3,-0.6 \mathrm{MPa}$. The results suggest that SwCNTs-treated soybean seeds showed improvement in tolerance to drought stress and the combined increases in SOD, CAT and POD activities in seeds may play an important role in the improvement of antioxidant capacity under drought stress.
\end{abstract}

Key words: Soybean, Germination percentage, Drought, Nanotubes.

\section{Introduction}

Achieving food security through a balance of resource management, environmental protection and sustainable agricultural development is important for food security (Ogbaji et al., 2013; Ogbaji et al., 2018; Shahrajabian et al., 2018; Yong et al., 2018; Shahrajabian et al., 2019a,b,c,d,e). Soybean is one of the world,s most important oil and protein crops (Murai et al., 2019). It is also 
one of the six most important crops in china. Drought in the sowing season can lead to the sowing time delay and reseed, through restraining seeds germination and grown. In china, drought and semiarid cultivated land takes up more than $40 \%$ and causes serious economic losses. Drought is the major obstacles to plant and production (Shahrajabian et al., 2013; Shahrajabian and Soleymani, 2017; Shahrajabian et al., 2017; Soleymani et al., 2017). Seed pretreatment with $\mathrm{H}_{2} \mathrm{O}_{2}$, $\mathrm{KNO}_{3}$, hydropriming, $\mathrm{GA}$, PEG, $\mathrm{KNO}_{3}-\mathrm{KH}_{2} \mathrm{PO}_{4}$, water has been confirmed to improve the germination and emergence in seeds of many crops under drought environments (Bian et al, 2013; Pipinis et al., 2017). Nanomaterial is an emerging substance and was widespread used in electron, medical treatment, aerospace, environment, agriculture because of the unique physicochemical property of it. Mariya Khodakovskaya, Alexandru Biris and their workmates in Arkansas university, firstly proved that the germination speed and sprout weight of tomato seeds which were treated with SwCNTs is more than double of the control. In the present study, we want to investigate SwCNTs function during germination and growth of soybean seeds under drought stress and discuss the putative mechanism of the action. Furthermore, the study examined the possibilities to overcome drought stress by seed pretreated treatment with SwCNTs.

\section{Materials and methods}

Soybean cultivar Zhonghuang 35, which is commonly grown in China, was used as seed material. To achieve drought condition, we treated soybean with polyethylene glycol (PEG 6000). Germination and early seeding growth (7 days) of the cultivar were studied under distilled water (control) and osmotic potentials of -0.3, -0.6, -0.9, -1.2 MPa for PEG 6000. SwCNTs was confected put up into solution 1 X 10-3 g/L.

\section{Seed treatments}

Five hundreds of uniformity plump seeds of soybean (Glycine max (L.) Merrill) Zhonghuang 35 were examined and surfaces-sterilized for $5 \mathrm{~min}$ in 5\% sodium hypochlorite solution, then rinsed thoroughly with deionized water sterilized. Seeds were split charging into two aseptic conical flasks with $100 \mathrm{~mL}$ SwCNTs solution and distilled water respectively. After 30 min poured-out liquor and dry the seeds on super clean bench.

\section{Germination tests}

Soybean seeds were placed on filter papers socked in $8 \mathrm{~mL}$ different concentrations of PEG 6000 in Petri dishes ( $9.0 \mathrm{~cm}$ diameter) and cultured at $26^{\circ} \mathrm{C}$ for 7 days in a growth chamber in the dark. There were 30 seeds in each Petri dish and the seeds were covered with aseptic gauzes dipped in different concentrations of PEG 6000. The gauzes were changed every day to keep the concentrations of solution. Seeds were considered to be germinated at the emergence of the 2 $\mathrm{mm}$ radicle and scored. After seven days, the germination was over and the germination, root length, shoot length and fresh weight were scored. The experiments were repeated three times.

\section{Activities of SOD, POD, CAT and contentions of MDA and $\mathrm{H}_{2} \mathrm{O}_{2}$ determination}

Activities of SOD, POD, CAT and contentions of MDA and $\mathrm{H}_{2} \mathrm{O}_{2}$ in SwCNTs-treated seeds and controls were determined respectively with the kits of SOD, POD, CAT, MDA and $\mathrm{H}_{2} \mathrm{O}_{2}$ produced by the Nanjing Jiancheng Bioengineering Institute (Nanjing, China). 


\section{Results}

\section{Effect of SwCNTs on seed germinability and germination percentage}

The results indicated that germinability and germination percentage was decreased with increase in osmotic potential in PEG solution. They are inhibited completely at -0.9 and -1.2 MPa of PEG. All the germinability and germination percentages in SwCNTs-treated seeds are more than control (Table 1).

Table 1. Germinability and germination percentage of seeds treated with SwCNTs and control at water stress of PEG

\begin{tabular}{|c|c|c|c|c|}
\hline \multirow{3}{*}{$\begin{array}{c}\text { PEG } \\
(\mathrm{MPa})\end{array}$} & \multicolumn{4}{|c|}{ Seed treatment } \\
\hline & \multicolumn{2}{|r|}{ SwCNTs } & \multicolumn{2}{|c|}{ Control } \\
\hline & $\begin{array}{c}\text { Germinability } \\
(\%)\end{array}$ & $\begin{array}{c}\text { Germination percentage } \\
(\%)\end{array}$ & Germinability & $\begin{array}{c}\text { Germination } \\
\text { percentage }(\%)\end{array}$ \\
\hline 0 & 95.6 & 97.8 & 93.3 & 95.6 \\
\hline-0.3 & 94.4 & 97.8 & 93.3 & 94.4 \\
\hline-0.6 & 48.9 & 83.3 & 47.8 & 80.0 \\
\hline-0.9 & - & - & - & - \\
\hline-1.2 & - & - & - & - \\
\hline
\end{tabular}

\section{Effect of SwCNTs on seed root and shoot length, fresh weight}

Although the root length of non-SwCNTs treated seeds were affected due to the water stress of PEG 6000, the seed treated with SwCNTs have significantly higher root length than control under osmotic potentials of $0,-0.3,-0.6 \mathrm{MPa}(\mathrm{P}<0.05 ; \mathrm{P}<0.01)$. The shoot length was also decrease with increase in osmotic potential in PEG solution. It were not evident at 0 and $-0.3 \mathrm{MPa}$, but it was evidently higher in SwCNTs treatment seeds than control at -0.6 $\mathrm{MPa}(\mathrm{P}<0.01)$. The variation trend of fresh weight was consistent with that of the root length, and the seed treated with SwCNTs have evident fresh weight than control under osmotic potentials of $0,-0.3,-0.6 \mathrm{MPa}(\mathrm{P}<0.05 ; \mathrm{P}<0.01)$ (Table 2$)$.

Table 2. Root length, shoot length and fresh weight of seeds treated with SwCNTs and control at water stress of PEG

\begin{tabular}{|c|c|c|c|c|c|c|}
\hline \multirow{3}{*}{$\begin{array}{c}\text { PEG } \\
(\mathrm{MPa})\end{array}$} & \multicolumn{6}{|c|}{ Seed treatment } \\
\hline & \multicolumn{2}{|c|}{ Root length (cm) } & \multicolumn{2}{|c|}{$\begin{array}{l}\text { Shoot length } \\
\text { (cm) }\end{array}$} & \multicolumn{2}{|c|}{$\begin{array}{c}\text { Fresh weight } \\
\text { (g.plant }^{-1} \text { ) }\end{array}$} \\
\hline & SwCNTs & Control & SwCNTs & Control & SwCNTs & Control \\
\hline 0 & $9.032^{* *}$ & $7.720^{* *}$ & 0.449 & 0.441 & $0.591^{* *}$ & $0.565^{* *}$ \\
\hline-0.3 & $8.806^{*}$ & 8.248* & 0.378 & 0.385 & $0.456^{*}$ & $0.421^{*}$ \\
\hline-0.6 & $6.774^{*}$ & $5.558^{*}$ & $0.229 * *$ & $0.209^{* *}$ & $0.366^{*}$ & $0.336^{*}$ \\
\hline-0.9 & - & - & - & - & - & - \\
\hline 1.2 & - & - & - & - & - & - \\
\hline
\end{tabular}




\section{Effect of SwCNTs on activities of SOD, POD, CAT and contents of $\mathrm{MDA}, \mathrm{H}_{2} \mathrm{O}_{2}$ during seed germination}

The contents of $\mathrm{H}_{2} \mathrm{O}_{2}$ in control seeds were decrease with the increase of the osmotic potentials and the activity of CAT had a slightly increase from 0 to -0.3 MPS then it declined by small margin at -0.6 MPS. It is confirmed that the content of $\mathrm{H}_{2} \mathrm{O}_{2}$ and the activity of CAT in SwCNTs-treated seeds had the contrary variation trend. The content of $\mathrm{H}_{2} \mathrm{O}_{2}$ increased slightly then declined trivially, but the activity of CAT was decreased with the increase of the osmotic potentials. Compare to control, the content of $\mathrm{H}_{2} \mathrm{O}_{2}$ descended and the activities of CAT increased significantly at 0 and -0.3 MPS (Figures 1,2 ). The activities of SOD and POD in the non-SwCNTs-treated seed had the homology trend that both of them showed the significant reduction with increase in osmotic potentials. In the SwCNTs-treated seed, the activity of SOD and POD also decrease with the increase of the osmotic potentials, but the activities at 0MPS were slightly below the control and the activities at $-0.3,-0.6$ MPS were advanced to the control (Figures 3,4). As the important sign of membrane-lipid peroxidation, MDA contents in SwCNTs-treated seeds were lower than control at -0.3, -0.6 MPS (Figure 5). The combined increase in SOD, CAT and POD activities in seeds plays an essential role in the improvement of antioxidant capacity at drought stress environment.

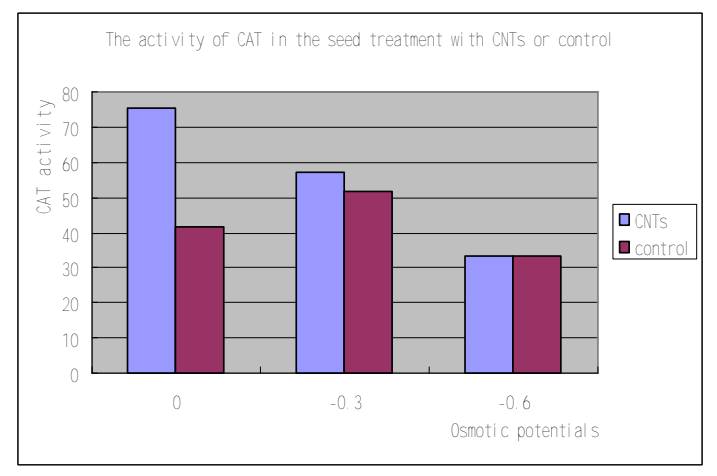

Figure 1. Effect of SwCNTs on the activity of CAT during seed germination. The activity of CAT of the controls were decreased with the increase of the osmotic potentials. The activity of CAT of SwCNTstreated seeds were decreased with the increase of the osmotic potentials but it were evidently higher than that of the controls under the osmotic potentials of $0,-0.3 \mathrm{MPa}$

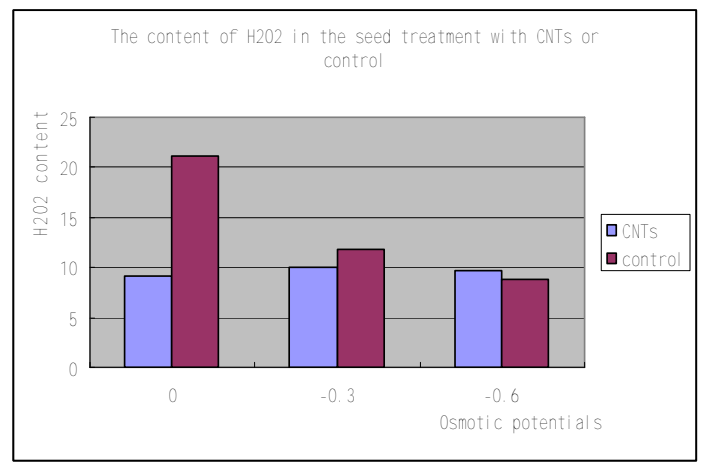

Figure 2. Effect of SwCNTs on the content of $\mathrm{H}_{2} \mathrm{O}_{2}$ during seed germination. The content of $\mathrm{H}_{2} \mathrm{O}_{2}$ of the controls were decreased with the increase of the osmotic potentials. The content of $\mathrm{H}_{2} \mathrm{O}_{2}$ of SwCNTs-treated seeds have little changes. It were increased slightly then declined trivially with the increase of the osmotic potentials 


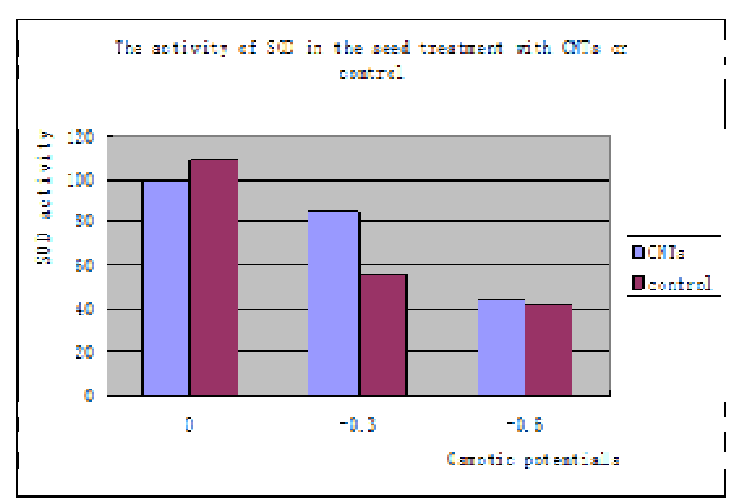

Figure 3. Effect of SwCNTs on the activity of SOD during seed germination. The activity of SOD of the controls were decreased with the increase of the osmotic potentials. The activity of SOD of SwCNTs-treated seeds were decreased too but it were evidently higher than that of the controls under the osmotic potentials of $-0.3,-0.6 \mathrm{MPa}$

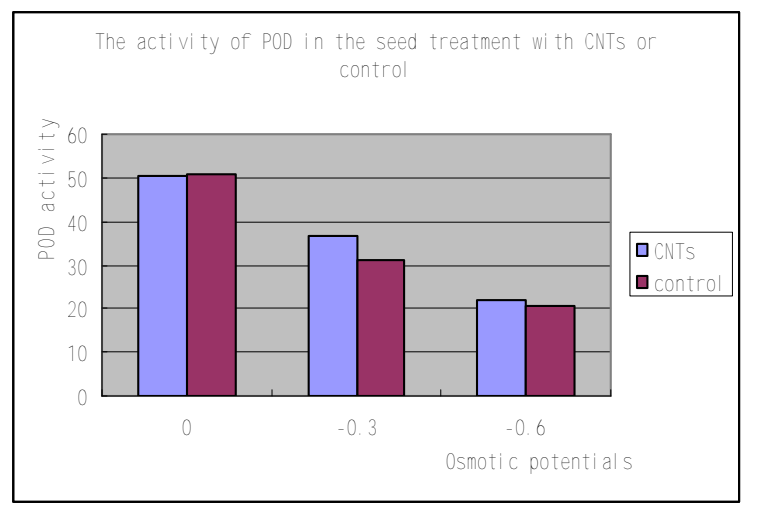

Figure 4. Effect of SwCNTs on the activity of POD during seed germination. The activity of POD of the controls were decreased with the increase of the osmotic potentials. The activity of POD of SwCNTs-treated seeds were decreased too but it were evidently higher than that of the controls under the osmotic potentials of $-0.3,-0.6 \mathrm{MPa}$

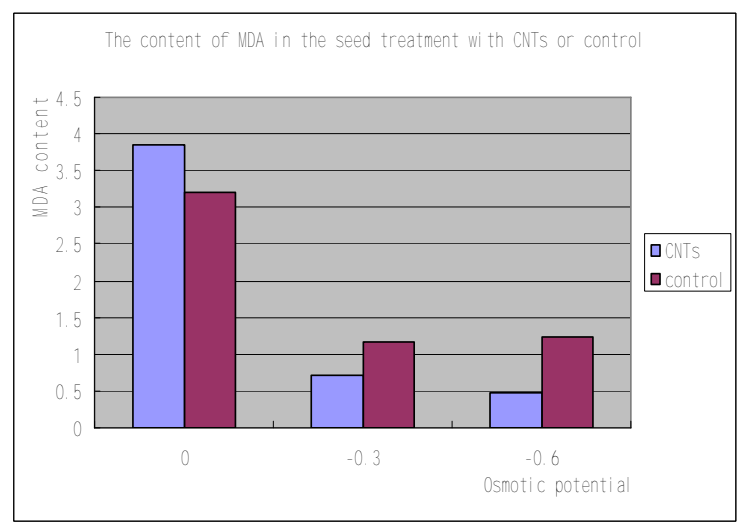

Figure 5. Effect of SwCNTs on the content of MDA during seed germination. The content of MDA of SwCNTs-treated seeds and that of the controls were decreased with the increase of the osmotic potentials. MDA content in the SwCNTs-treated seed was lower than control at -0.3, --0.6 MPS 


\section{Discussion}

Drought is the major reason to yield losing of soybean. Our study indicated that although the water stress condition delayed the germination considerably, seed pretreatment with SwCNTs can improve the germination percentage significantly. Fresh weights of SwCNTs treated seeds were evident higher than control under osmotic potentials of $0,-0.3,-0.6 \mathrm{MPa}$ $(\mathrm{P}<0.05 ; \mathrm{P}<0.01)$. Root length was longer than control under osmotic potentials of $0,-0.3$, $-0.6 \mathrm{MPa}(\mathrm{P}<0.05 ; \mathrm{P}<0.01)$. Shoot length was significantly higher in the SwCNTs treatment than control at $-0.6 \mathrm{MPa}(\mathrm{P}<0.01)$. The result supports that SwCNTs can improve the germination of soybean under drought stress condition. The carbon treatment did not improve the germination of soybean seeds (date not shown), so the structure of SwCNTs may play an important role in the case. The SwCNTs might penetrate the hard seed coat of soybean. The positive effect of SwCNTs to the germination of soybean seed might be explained by more rapid and enhanced water uptake effect. The production of AOS by germinating seeds has often been regarded as a cause of stress that might affect the success of germination (Christophe Bailly, 2004). Loss of viability during drying of recalcitrant seeds of Quercus robur (Finch-Savage et al., 1994), Shorea robusta (Chaitanya and Naithani, 1994) and Theobroma cocoa (Li and Sun, 1999) is accompanied by a loss of the cellular antioxidant potential and an accumulation of free radicals. So the ability of cells to withstand loss of water might be closely related to AOS scavenging (Christophe Bailly, 2004). Protection against oxidative stress during imbibition has also been suggested for peroxiredoxins. Our results suggest that the contention of $\mathrm{H}_{2} \mathrm{O}_{2}$ in SwCNTs-treated seed was less than the control and activities of CAT, SOD, POD in SwCNTs-treated seeds were more than the control. Meanwhile, the MDA content in SwCNTs-treated seeds was lower than control at $-0.3,-0.6$ MPS. The results are in line with the findings of the resurrection plant Xerophyta viscose (Sherwin and Farrant, 1998), and germinated maize (Leprince et al., 1990) or wheat seeds (Farrant et al., 2004). The germination of soybean under drought stress is also associated with changes in the balance of AOS content and detoxifying enzyme activities. The SwCNTs can promote the germination of soybean seed against desiccation stress and it may rapidly induce or enhance the cell signaling. This may lead to the expression of many genes, including those coding for defense-related proteins, transcription factors, phosphatases, kinases and enzymes involved in AOS synthesis or degradation.

\section{Conclusion}

Our results suggest that the SwCNTs promote the germination of soybean seed under drought stress significantly and it also decrease the contents of $\mathrm{H}_{2} \mathrm{O}_{2}$ and MDA. Meanwhile, it increased activities of CAT, SOD and POD in soybean seeds during early seed inhibition. This research may provide a new way of seed priming in soybean to overcome drought stress during germination and it also gave a foundation to the study of the mechanism of the SwCNTs to the soybean seed germination.

\section{References}

Bian, L, Yang, L, Wang, JA, Shen, HL. (2013). Effects of $\mathrm{KNO}_{3}$ pretreatment and temperature on seed germination of Sorbus pohuashanensis. J. Forestry Res., 24(2):309-316. 
Christophe B. (2004). Active oxygen species and antioxidants in seed biology, Seed Sci. Res., 14:93-107.

Chaitanya, KSK, Naithani, SC. (1994). Role of superoxide, lipid peroxidation and superoxide dismutase Active oxygen species and antioxidants in seeds 103 in membrane perturbation during loss of viability in seeds of Shorea robusta Gaerth. New Phytol., 126:623-627.

Farrant, JM, Bailly, C, Leymarie, J, Hamman, B, Côme, D, Corbineau, F. (2004). Wheat seedlings as a model to understand desiccation-tolerance and sensitivity. Physiol. Plant., 120:563-574.

Finch-Savage, WE, Hendry, GAF, Atherton, NM. (1994). Free radical activity and loss of viability during drying of desiccation sensitive tree seeds. Proceed. Royal Soc. Edinb., 102B:257-260.

Leprince, O, Deltour, R, Thorpe, PC, Atherton, NM, Hendry, GAF. (1990) The role of free radicals and radical processing systems in loss of desiccation tolerance in germinating maize (Zea mays L.). New Phytol., 116:573-580.

Li, C, Sun, WQ. (1999) Desiccation sensitivity and activities of free radical-scavenging enzymes in recalcitrant Theobroma cacao seeds. Seed Sci. Res., 9:209-217.

Murai, Y, Takahashi, R, Kitajima, J, Iwashina, T. (2019). New quercetin triglycoside from the leaves of soybean cultivar Clark. Natural Product Commun., 14(5):1934578X1984361.

Ogbaji, PO, Shahrajabian, MH, Xue, X. (2013). Changes in germination and primarily growth of three cultivars of tomato under diatomite and soil materials in auto-irrigation system. Int. J. Biol., 5(3):80.

Ogbaji, PO, Li, J, Xue, X, Shahrajabian, MH, Egrinya Eneji, A. (2018). Mineralogical and textural characteristics of soils of Hancheng and Shannxi province, China. Commun. Soil Sci. Plant Anal., 49(3):1-5.

Pipinis, E, Stampoulidis, A, Milios, E, Kitikidou, K, Radoglou, K. (2017). Effects of cold stratification and GA3 on germination of Arbutus Unedo seeds of three provenances. Afr. J. Tradit., Complement. Altern. Med., 14(1):318-323.

Shahrajabian, MH, Xue, X, Soleymani, A, Ogbaji, PO, Hu, Y. (2013). Evaluation of physiological indices of winter wheat under different irrigation treatments using weighing lysimeter. Int. J. Farm. Allied Sci., 2(24):1192-1197.

Shahrajabian, MH, Soleymani, A. (2017). A lysimeter study, a unique tool for botanists, agronomists and other plant scientists. Asian Res. J. Agric., 4(2):1-9.

Shahrajabian, MH, Soleymani, A, Ogbaji, PO, Xue, X. (2017). Impact of different irrigation managements on soil water consumption, grain yield, seed protein, phosphorus and potassium of winter wheat. Cercet. Agronom. Mol., 3(171):5-13.

Shahrajabian, MH, Sun, W, Cheng, Q. (2018). A review of Goji berry (Lycium barbarum) in traditional Chinese medicine as a promising organic superfood and superfruit in modern industry. Academia J. Med. Plant., 6(12):437-445. 
Shahrajabian, MH, Sun, W, Cheng, Q. (2019a). The power of natural Chinese medicine, ginger and ginseng root in an organic life. Middle-East J. Sci. Res., 27(1):64-71.

Shahrajabian, MH, Sun, W, Cheng, Q. (2019b). Clinical aspects and health benefits of ginger (Zingiber officinale) in both traditional Chinese medicine and modern industry. Acta Agric. Scandin., Section B-Soil Plant Sci., 69(6):1-11.

Shahrajabian, MH, Sun, W, Cheng, Q. (2019c). Traditional Chinese medicine and agriculture; organic life and sustainability for future. GSCBiol. Pharm. Sci., 7(01):091-095.

Shahrajabian, MH, Sun, W, Cheng, Q. (2019d). A review of ginseng species in different regions as a multipurpose herb in traditional Chinese medicine, modern herbology and pharmacological science. J. Med. Plants Res., 13(10):213-226.

Shahrajabian, MH, Khoshkharam, M, Sun, W, Cheng, Q. (2019e). The effect of pretreatment factors on seed germination and seedling growth of anise (Pimpinella anisum L.). Middle-East J. Sci., 5(1):86-93.

Sherwin, HW, Farrant, JM. (1998). Protection mechanisms against excess light in the resurrection plants Craterostigma wilmsii and Xerophyta viscosa. Plant Growth Regulat., 24:203-210.

Soleymani, A, Shahrajabian, MH. (2017). Assessment of ET-HS model for estimating crop water demand and its effects on yield and yield components of barley and wheat in semi-arid region of Iran. Cercet. Agron. Mol., 4(172):37-49.

Yong, Y, Hu, YG, Shahrajabian, MH, Ren, CZ, Guo, LC, Wang, CL, Zeng, ZH. (2018). Changes in dry matter, protein percentage and organic matter of soybean-oat and groundnut-oat intercropping in different growth stages in Jilin province, China. Acta Agric. Slovenica, 111(1):17.

How to cite this article: Sun Wenli, Mohamad Hesam Shahrajabian, Qiman Huang. Soybean Seeds Treated with Single Walled Carbon Naotubes (SwCNTs) Showed Enhanced Drought Tolerance During Germination. International Journal of Advanced Biological and Biomedical Research, 2020, 8(1), 9-16. Link: http://www.ijabbr.com/article 36169.html 